\title{
IPILIMUMAB-INDUCED HYPOPHYSITIS IN CANCER PATIENTS
}

AUTHORS: Christian Lafuente1, Karina Arcano1, Fernando Guerrero², Agustina Pia Marengo ${ }^{2}$; Inmaculada Peiró ${ }^{2}$, Ainara Soria ${ }^{3}$, Juan José Diez ${ }^{1}$, Carles Villabona ${ }^{2}$ and Pedro Iglesias ${ }^{1}$.

AFFILIATIONS: Departments of ${ }^{1}$ Endocrinology and ${ }^{3}$ Oncology, Hospital Ramón y Cajal, Madrid, Spain and Department of ${ }^{2}$ Endocrinology, Hospital Bellvitge, Barcelona, Spain.

CONTEXT: Ipilimumab is a human monoclonal antibody against cytotoxic T lymphocyte antigen-4 (CTLA-4), which enhances stimulation of cytotoxic $T$ lymphocytes resulting in an immune response against the tumor. This drug-induced hyperactivity of the immune system can lead to serious adverse effects including endocrine disorders such as autoimmune hypophysitis, thyroiditis and adrenal insufficiency.

OBJECTIVE: To report our experience on ipilimumab-induced hypophysitis (IIH) in patients with advanced cancer.

RESULTS: Four patients [3 men (75\%); aged $67.5 \pm 8.7$ years (range, 59-75)] with advanced (stage IV) cancer, (3 melanomas and one prostate cancer) were recruited (table 1 and figure 1). The clinical presentation of IIH was similar in all cases regardless of the underlying tumor. IHH developed after 10-20 weeks of starting treatment, usually between 2 and 4 ipilimumab cycles. Main symptoms were fatigue $(n=3)$ and headache $(n=2)$, nausea $(n=2)$ and vomiting $(n=2)$. IIH was associated with partial or total hypopituitarism in 3 cases. Pituitary hormone deficiencies most frequently affected were corticotrophin $(A C T H, n=3)$, thyrotropin $(T S H$, $\mathrm{n}=3$ ) and gonadotropins ( $\mathrm{FSH}$ and $\mathrm{LH}, \mathrm{n}=3$ ). Two patients showed prolactin and growth hormone deficiencies. One patient did not develop any pituitary hormone deficiency although magnetic resonance imaging (MRI) of the pituitary showed morphologic changes of hypophysitis. MRI study showed enlarged pituitary in 3 patients. All patients were treated with high steroid doses (>0.5 mg/kg/day of prednisone or equivalent). After $22.1 \pm$ 13.8 months (range, 3.9-37.6) of follow-up from the first dose of ipilimumab, 3 patients remained with steroid hormone replacement therapy and 2 patients with replacement doses of levothyroxine. One patient recovered thyroid function.
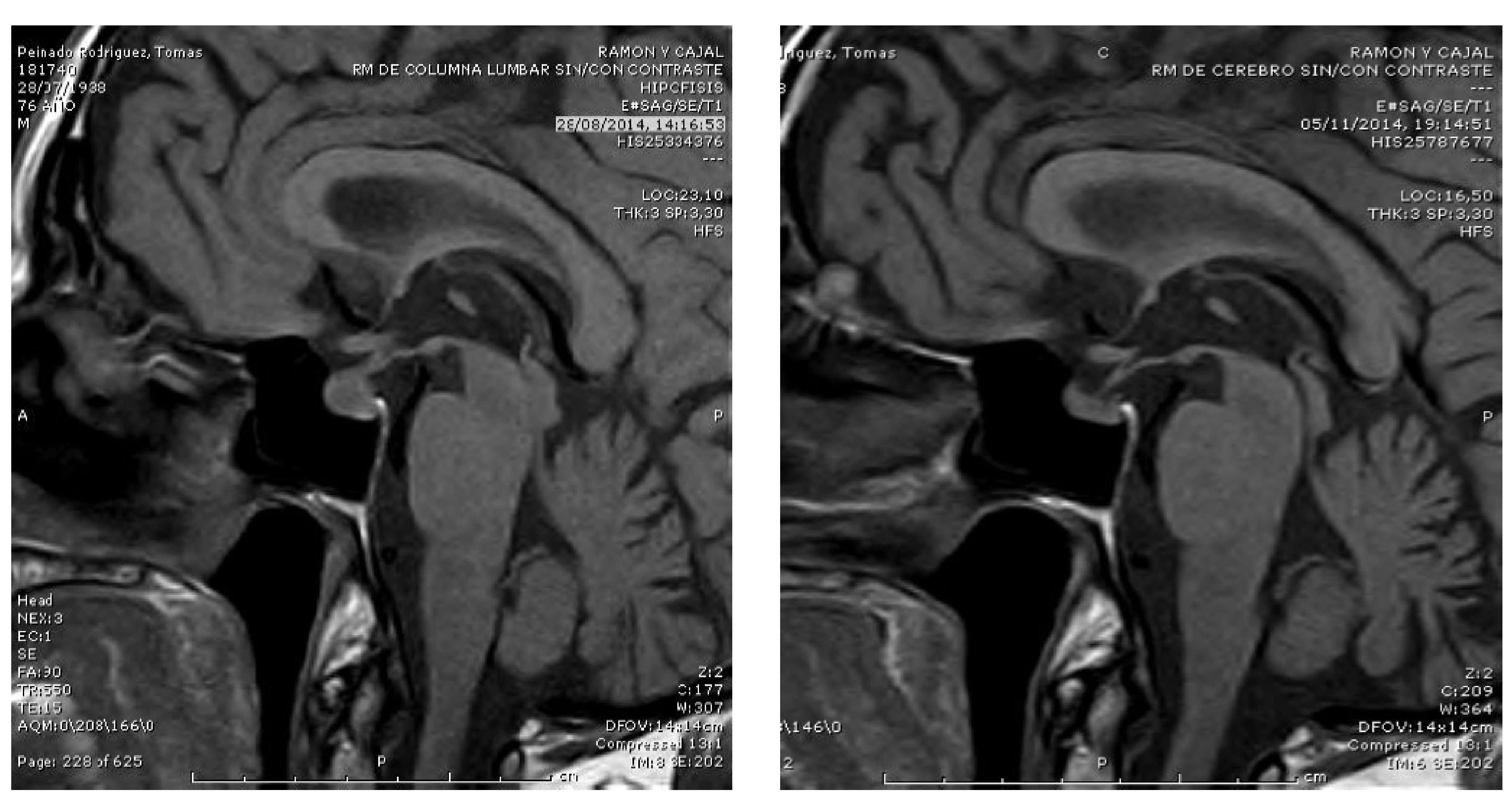

Figure 1. MRI showing enlarged pituitary gland and thickening of the pituitary stalk at IIH diagnosis (left) and three months after starting therapy (right) (patient \# 3).
Table 1. Clinical and analytical features of the four patients with ipilimumab-induced hypophysitis.

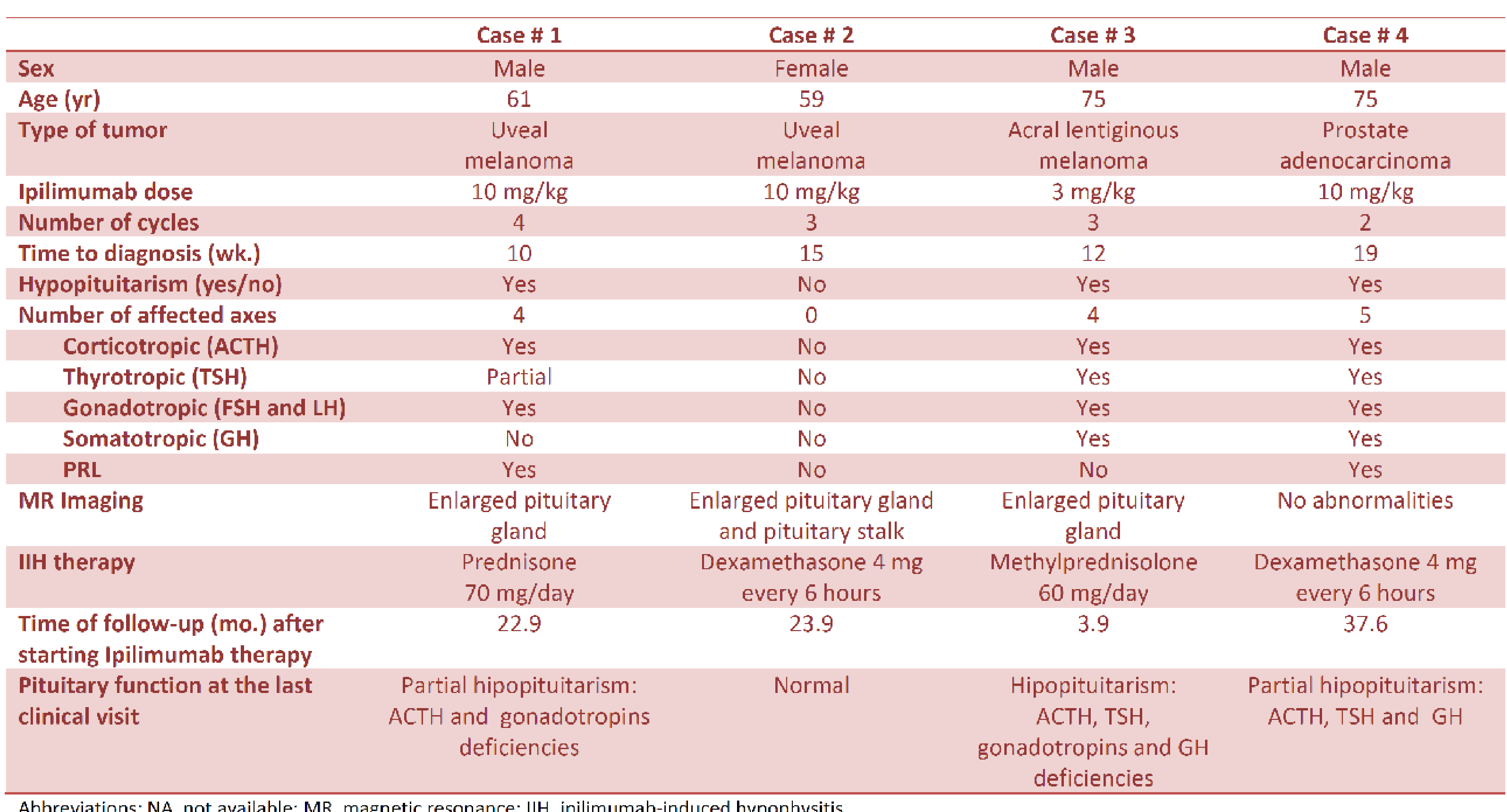

CONCLUSION: IIH is an immune-related adverse event that can compromise the patient's life because of the possibility of development of adrenal and thyroid insufficiency in a high percentage of patients. Glucocorticoids are the treatment of choice. Hormone replacement therapy is indicated according to hormone deficiencies. Patients receiving immunomodulatory therapies should be closely monitored by baseline and follow-up hormone assessment. 